\title{
Actitudes de los estudiantes sin discapacidad con los compañeros con discapacidad del centro regional de lbagué en la Universidad Nacional Abierta y a Distancia UNAD-Colombia.
}

\author{
Luigi López ${ }^{1}$ \\ Gloria Isabel Vargas Hurtado ${ }^{2}$ \\ Nancy Edith Ochoa Guevara ${ }^{3}$
}

\section{Resumen}

Este artículo tiene como objeto mostrar los resultados del estudio de las actitudes que tienen los estudiantes sin discapacidad hacia su compañeros con algún tipo de discapacidad en el marco del proyecto de investigación Sistema de Información para la Población con alguna Discapacidad (Proyecto SIDIS). La población estudiada fueron los estudiantes sin discapacidad de las escuelas y programas académicos de: Escuela de Ciencias Básicas, Tecnología e Ingeniería (Ingeniería de Sistemas, Ingeniería Industrial). Escuela de Ciencias Administrativas, Contables Económicas y de Negocios (Administración de Empresas). Escuela de Ciencias Agrícolas, Pecuarias y del Medio Ambiente (Zootecnia, Ingeniería Ambiental, Agronomía, Ingeniería Agroforestal). Escuela de Ciencias de la Educación (Licenciatura en Inglés). Escuela de Ciencias Sociales, Artes y Humanidades (Psicología, Comunicación Social), perteneciente al Centro Regional (CEAD) de Ibagué de la Universidad Nacional Abierta y a Distancia - UNAD, Colombia. La actitud arrojada en la investigación fue en general favorable, sin embargo no se puede generalizar a todos los centros regionales de la UNAD, ni a toda la población estudiantil sin discapacidad del centro regional de estudio, ya que este es solo una aproximación a las actitudes que presentan los estudiantes que tienen compañeros con discapacidad. Esta investigación fue realizada por estudiantes de doctorado en la UNAD -Florida (EE.UU).

Palabras clave: actitudes, discapacidad, inclusión educativa, limitación física. 


\title{
Attitude of students without disabilities toward handicapped students. Open National University and at Distance - UNAD. Colombia. Regional center lbague.
}

\begin{abstract}
This article shows the results of the study about the attitudes of students without disability have towards their classmates with some type of disability, within the research project System of Information for Population with some Disability (SIDIS Project).

The population studied included students without disabilities from the following Schools and Programs: School of Basic Sciences, Technology and Engineering (Systems Engineering, Industrial Engineering); School of Administrative, Economic, Accountant and Business Sciences (Business Administration); School of Agricultural, Cattle and Environmental Sciences (Zootecnnics, Environmental Engineering, Agronomy, Agroforestry Engineering); School of Educational Sciences (Bachelor in English); School of Social Sciences, Arts and Humanities (Psychology, Social Communication), belonging to Ibagué Regional Center (CEAD) from Open and Distance National University - UNAD, Colombia.

The attitude found in this study was overall favorable. However this can not be generalized to all UNAD regional centers, neither to the whole student population without disability from the regional center due to this study is just an approach to the attitudes from students who have classmates with limitation.
\end{abstract}

Key words: attitudes, disability, educational inclusion, physical limitation.

Recibido: 11 de julio de 2013

Aceptado: 26 de septiembre de 2013 


\section{lntroducción}

Es ahora cuando se ha estimulado un poco más el tema de la inclusión de las personas con discapacidad en todos los ámbitos de la vida: educativo, social, cultural, deportivo y laboral, con esto se busca la igualdad de condiciones sociales, así como mejorar su calidad de vida, desde el punto de vista formativo, económico, cultural e interdisciplinaria, como es el caso de la inclusión educativa y social.

Para que ocurra la inclusión y las personas con discapacidad gocen de plena libertad de elegir una carrera y una universidad, es necesario que se adopten medidas en cuanto a la igualdad de oportunidades, la no discriminación, las ofertas de programas académicos y la infraestructura, entre otras: En cuanto a la infraestructura, la mayoría de las instituciones de educación superior no tienen condiciones óptimas de accesibilidad para el ingreso y movilidad de las personas con discapacidad. Así mismo es necesario adoptar medidas para informar a la población en general sobre las personas con discapacidad, tales como campañas o charlas de concientización sobre la importancia de involucrar a estas personas en el entorno educativo y social.

La presente investigación trata el tema de las personas con discapacidad desde el punto de vista educativo, tomando en cuenta las actitudes que asumen los estudiantes sin discapacidad ante sus compañeros que presenten alguna discapacidad notoria (ya sea sensorial a nivel visual o auditiva, física o motora, e intelectual o mental ), en el Centro Regional (CEAD) de Ibagué de la Universidad Nacional Abierta y a Distancia -UNAD - Colombia, ya que según las teorías de la actitud, son éstas las que definen nuestras conductas y acciones, y por ende la implementación, ejecución, cumplimiento y apoyo a las leyes y normativas que determinan la participación educativa y social de las personas con discapacidad.

Dentro de los factores determinantes del éxito de la atención a la diversidad, la actitud favorable de los agentes implicados en el proceso, aparece como uno de los más importantes. El concepto de actitud ha sido ampliamente estudiado y ha dado lugar a numerosos estudios, sobre todo en la Psicología Social. Conocer qué son las actitudes, cómo se forman, cómo se pueden medir y modificar, son interrogantes que han despertado el interés científico desde hace mucho tiempo.

Las actitudes pueden ser desfavorables o favorables, si existe una actitud desfavorable, la persona tiende a no aceptar a las personas con discapacidad, mientras que con una actitud favorable ocurre lo contrario, es decir, se tiende a la aceptación de las personas con discapacidad. Para la realización del trabajo se tomó como muestra las personas sin discapacidad que estudian en el CEAD de Ibagué en la UNAD, Colombia, que tuvieran compañeros en su aula de 
clase presencial con discapacidad. Una vez obtenidos los datos, se procedió al análisis de los mismos.

En la realización de la investigación se recurrió a trabajos realizados sobre el tema en Colombia, así como en otros países: Venezuela, España, Canadá, Chile y Argentina; artículos de prensa, e información suministrada por diversos organismos encargados de la inclusión social y educativa de las personas con discapacidad. Se tomó como referencia las actitudes de los estudiantes sin discapacidad hacia sus compañeros con discapacidad en el aula de clase presencial y en general al contexto educativo y al universitario en particular.

El estudio de las actitudes de las personas sin discapacidad hacia las personas con discapacidad es de gran importancia, sin embargo hay pocas investigaciones que precisen este tema, como lo sugieren Verdugo, Jenaro y Arias:

“... a pesar de la importancia primordial de las actitudes hacia las personas con discapacidad para lograr una integración social real, todavía son pocos los centros e instituciones que incluyen, como parte importante de su quehacer profesional, actividades, objetivos y contenidos dirigidos a evaluar y mejorar las actitudes”, (1994).

Es por esto que se considera que esta investigación es innovadora y trascendente, reiterando el respeto a la diversidad humana, como bien lo indica Aramayo expresando que:

La diversidad caracteriza al ser humano. Cada persona es única y diferente a las demás. No existe nada igual ni homogéneo en el hombre, en su aspecto físico, cualidades intelectuales, sus sentimientos, emociones $u$ otras dimensiones. Con razón se exige respeto a la diversidad de género, color, etnia, religión, cultura. ¿Qué pasa con la discapacidad, si ésta es sólo una forma de diversidad, posiblemente, más dramática? (2005: 145).

El propósito del estudio es conocer las actitudes de los estudiantes sin discapacidad hacia los compañeros con discapacidad en la clase presencial en el CEAD de Ibagué en la UNAD. Para este trabajo se parte de la hipótesis de que los jóvenes estudiantes del CEAD de diferentes escuelas y programas académicos de la UNAD, muestran una actitud favorable hacia la inclusión de sus compañeros con discapacidad. Identificando las variables que determinan precisamente la existencia de una actitud favorable hacia la discapacidad de sus compañeros de aula.

Por último se muestran los resultados del estudio enfocados en dar solución a la hipótesis y objetivos trazados desde su inicio al igual que las conclusiones y recomendaciones para tener en cuenta en próximas investigaciones acorde al tema de trabajo. 


\section{Metodología}

En este estudio se aplicó una metodología cuantitativa, ya que se buscaba "cuantificar y aportar evidencia a una teoría que se tiene para explicar algo (...)" (Gómez, 2006: 61). Utilizando el proceso de investigación descriptiva tratando de analizar y responder la hipótesis de que los jóvenes estudiantes del CEAD de diferentes escuelas y programas académicos de la UNAD, muestran una actitud favorable hacia sus compañeros con algún tipo de discapacidad.

La investigación es de tipo transversal y correlacional, ya que los datos fueron recabados en un sólo momento histórico, los resultados obtenidos fueron sometidos a un análisis y de este modo se identificó y describieron las variables (Hernández et al., 2006), es decir, se alcanzaron los resultados producto de la recolección de los datos en un sólo momento.

El diseño del presente estudio es no experimental, debido a que no se manipula la variable independiente, pues ya ocurrió, y los sujetos ya pertenecían a un grupo o nivel determinado de la variable independiente por auto selección (Gómez, 2006: 102).

Se realizó una encuesta a 75 jóvenes matriculados en el Centro Regional de Ibagué de la UNAD, en diferentes escuelas y programas académicos. El estudio fue realizado en el mes de abril de 2013. El proceso de selección de la muestra priorizó la participación de aquellos estudiantes con compañeros con alguna discapacidad. La razón de esta selección intencional está motivada porque se trata de conocer las actitudes de estos estudiantes sin discapacidad que han tenido mayor oportunidad de relacionarse con estudiantes con discapacidad.

Con base en la información aportada por la oficina de Registro y Control del Centro Regional de Ibagué se tomó en cuenta la siguiente información: nombre de la escuela, nombre programas académicos, lista de estudiantes que podían acceder al estudio, y ademas se escogió una muestra de cinco (5) escuelas con diez (10) programas académicos respectivamente. Escuela de Ciencias Básicas, Tecnología e Ingeniería - ECBTI. (Programas: Ingeniería de Sistemas, Ingeniería Industrial). Escuela de Ciencias Administrativas, Contables Económicas y de Negocios ECACEN. (Programas: Administración de Empresas). Escuela de Ciencias Agrícolas, Pecuarias y del Medio Ambiente ECAPMA. (Programas: Zootecnia, Ingeniería Ambiental, Agronomía, Ingeniería Agroforestal). Escuela de Ciencias de la Educación ECEDU (Licenciatura en Inglés) y Escuela de Ciencias Sociales, Artes y Humanidades ECSAH. (Programas: Psicología, Comunicación Social). 


\section{lnstrumento}

Previamente a la realización del trabajo de campo, se diseñó un cuestionario entre los investigadores el cual fue analizado, valorado y depurado por un experto en el tema. La variable que se midió en el estudio fue la actitud de los estudiantes sin discapacidad ante sus compañeros de aula con discapacidad. Este instrumento se aplicó a una muestra de 75 estudiantes.

Para la recolección de los datos se usó un cuestionario de escalas de actitudes tipo Likert en la que se valoraba el grado de acuerdo o desacuerdo en una escala de 1 (nada de acuerdo) a 5 (totalmente de acuerdo), con una serie de reactivos que surgieron de la variable actitud.

\section{Procedimientos}

La información obtenida a través de los diferentes instrumentos y procedimientos utilizados, se analizó y se redujo en función del tópico de la investigación, estableciendo categorías para su posterior análisis. Como variable dependiente se destaca la actitud de los estudiantes frente a sus compañeros de aula, mientras que la variable independiente seria la escuela y el programa académico a donde están inscritos dichos estudiantes.

En la tabla 1, se observa los elementos incluidos en la operacionalización de la variable del estudio logrando conocer la magnitud del tipo de actitud de los estudiantes hacia sus compañeros en condición de discapacidad.

Tabla 1. Operacionalización de la variable actitud.

\begin{tabular}{|c|c|c|c|}
\hline Variable & Definición & Actitudes & $\begin{array}{l}\text { Ítem asociados a los } \\
\text { indicadores }\end{array}$ \\
\hline \multirow{3}{*}{$\begin{array}{l}\text { Actitud hacia las } \\
\text { personas con } \\
\text { discapacidad }\end{array}$} & \multirow{3}{*}{$\begin{array}{l}\text { Conjunto de creencias, } \\
\text { juicios, emociones, } \\
\text { prejuicios, estereotipos } \\
\text { y conductas que } \\
\text { poseen } \\
\text { los individuos hacia las } \\
\text { personas con } \\
\text { discapacidades. } \\
\text { Dichas } \\
\text { actitudes son } \\
\text { construidas por } \\
\text { diversos } \\
\text { factores externos al } \\
\text { individuo }\end{array}$} & $\begin{array}{c}\text { Actitud ante } \\
\text { Competencias } \\
\text { cognitiva (Aprendizaje, } \\
\text { enseñanza- } \\
\text { aprendizaje) }\end{array}$ & $\begin{array}{c}1,2,3,4,5,6,7,8 \\
9,10\end{array}$ \\
\hline & & $\begin{array}{l}\text { Actitud ante condición } \\
\text { física }\end{array}$ & $\begin{array}{c}11,12,13,14,15,16 \\
17,18,19,20\end{array}$ \\
\hline & & $\begin{array}{l}\text { Actitud ante } \\
\text { Competencia afectiva } \\
\text { (Relaciones ínter e } \\
\text { intra personales) }\end{array}$ & $\begin{array}{c}21,22,23,24,25,26 \\
27,28,29,30\end{array}$ \\
\hline
\end{tabular}

Fuente: autores.

Con la finalidad de lograr el registro de los resultados en el programa estadístico SPSS 12.0, se creó una serie de códigos a fin de facilitar la posterior tabulación de estos resultados. En la tabla 2, se aprecia los códigos asignados a las opciones de respuesta siguiendo la escala de Likert. 
Tabla 2. Códigos de las respuestas del instrumento aplicado.

\begin{tabular}{|c|c|}
\hline Código & \begin{tabular}{c} 
Opciones de respuesta \\
\hline 5
\end{tabular} \\
\hline 4 & TA = Totalmente de Acuerdo \\
\hline 3 & $\mathrm{DA}=$ De Acuerdo \\
\hline 2 & $\mathrm{NDA}=\mathrm{Ni}$ de Acuerdo Ni en Desacuerdo (Indiferente) \\
\hline 1 & $\mathrm{ED}=$ En Desacuerdo \\
\hline
\end{tabular}

Fuente: autores.

El instrumento fue sometido a la evaluación de validez y confiabilidad ${ }^{4}$ a través de una prueba piloto ingresando todos los datos por el SPSS ver 12.1.0, con el fin de calcular el Alfa Cronbach ${ }^{5}$ con un porcentaje de $0.716 \%$ lo cual permitió continuar con el estudio y tener un grado aceptable del instrumento de medición.

En la tabla 3, se observa la codificación que se realizó en la escala de la variable de actitud del estudio en donde se resume la interpretación de los puntajes obtenidos por los estudiantes sin discapacidad en la encuesta sobre la actitud hacia las personas con discapacidad.

Tabla 3. Codificación y escala para la variable del estudio.

\begin{tabular}{|c|c|c|}
\hline $\begin{array}{c}\text { Codificación de } \\
\text { los Puntajes }\end{array}$ & Rango de los Puntajes & \begin{tabular}{c} 
Interpretación del puntaje obtenido \\
\hline 5
\end{tabular} \\
\hline 4 & 0,81 a 1,00 & Actitud muy favorable \\
\hline 3 & 0,61 a 0,80 & Actitud bastante favorable \\
\hline 2 & 0,41 a 0,60 & Actitud indeciso o neutro \\
\hline 1 & 0,21 a 0,40 & Actitud bastante desfavorable \\
\hline
\end{tabular}

Fuente: autores.

\section{Análisis de la información y Resultados}

El análisis de los datos se llevó a cabo a través de la estadística descriptiva para estadística descriptiva para ordenar y analizar los datos con el fin de describir la muestra, ya que no se manipulo ninguna variable.

Se tomó como base el análisis descriptivo univariado, puesto que existe únicamente una variable dependiente (actitud hacia los estudiantes en condición de discapacidad en el aula de estudio) y se compararon los resultados obtenidos

4 El Alfa Cronbach promedio está basado en los autovalores promedio.

5 Nivel de confianza $70 \%$, probabilidad p . $q=50 \%$, error $10 \%$. 
por los diferentes grupos de la variable independiente (escuela de la UNAD a través de los programas académicos).

En general, los datos obtenidos muestran una puntuación media aproximada de un 3,8 en una escala que tiene un valor mínimo de 1 (totalmente en desacuerdo) y máximo de 5 (totalmente de acuerdo), por lo que podemos decir que la muestra analizada presenta una actitud favorable hacia los compañeros en condición de discapacidad.

En la Tabla 4, se puede observar que la media de los estudiantes de la Escuela de Sociales Artes y Humanidades es la mayor, siendo similares las del resto de las carreras. Por su parte, en la Escuela de Ciencias Básicas e Ingeniería, la media es una de las más bajas pero con una desviación típica bastante alta. Así pues, se valida parcialmente la hipótesis del estudio: las actitudes de los estudiantes sin discapacidad es favorable hacia sus compañeros con discapacidad mostradas por los estudiantes de la Escuela de Sociales Artes y Humanidades y la Escuela de Ciencias Básicas, Tecnología e Ingeniería que en las demás escuelas de la Universidad. Escuela de Ciencias Agrícolas, Pecuarias y del medio Ambiente Escuela de Ciencias de la Educación

Tabla 4. Análisis descriptivo variable independiente (Escuelas).

\begin{tabular}{|c|c|c|c|}
\hline & $\mathbf{N}$ & Media & Desviación típica \\
\hline ECBTI & 14 & 3.69 & 0.52 \\
\hline ECSAH & 12 & 4.27 & 0.24 \\
\hline ECACEN & 17 & 3.8 & 0.39 \\
\hline ECAPMA & 17 & 3.5 & 0.39 \\
\hline ECEDU & 15 & 3.8 & 0.37 \\
\hline TOTAL & 75 & 3.8 & 0.45 \\
\hline
\end{tabular}

Fuente: autores.

Para realizar el contraste de hipótesis, se llevó a cabo una prueba paramétrica a partir de la cual se comparan los distintos estudiantes de los programas académico. Esto es posible porque se cumplen los requisitos de distribución normal, homocedasticidad y escala de medida de intervalo.

\section{Discusión}

Para realizar la recolección de datos se creó un instrumento de medición (cuestionario) referente a las actitudes de los estudiante sin discapacidad hacia sus compañeros de aula con discapacidad.

Este instrumento se envió por el correo algunos estudiantes y a otros se les aplicó directamente en el Centro Regional con el apoyo de algunos docentes. Un total de 75 estudiantes del centro regional de Ibagué contestaron voluntariamente el cuestionario, puntuando en una escala del 1 al 5. 
En cuanto a los resultados obtenidos, se puede afirmar que la hipótesis del estudio se cumple parcialmente, ya que las puntuaciones en la ECSAH son las más positivas; sin embargo, en la ECBTI muestra una de las puntuaciones más bajas, aunque con mayor dispersión.

Por una parte, estos resultados pueden deberse a que la muestra obtenida es representativa en cuanto a la población del centro regional, pero no en cada escuela académica de la universidad.

Por otra parte, sorprende el hecho de que la media de la ECBTI sea una de las más bajas en comparación con las demás. Esto podría hacer pensar que es necesaria una revisión al interior de la escuela. Sin embargo desde la experiencia de los investigadores se concretó a través de la observación que en ella se contempla la atención hacia la población en condición de discapacidad desde una perspectiva positiva. Así pues, se deduce que existen factores ajenos a la formación académica que también influyen sobre las actitudes, como la familia, el entorno social, los medios de comunicación.

A partir de esta investigación, a pesar de que los resultados muestran que las actitudes hacia la los estudiantes en condición de discapacidad, en general, han salido positivas, se cree que sería interesante ofrecer algunos talleres o diplomados en el ámbito social transversal a todas las escuelas sobre el tema. Se considera que existen elementos que se pueden tener en cuenta en la realización de posteriores investigaciones, como por ejemplo la selección de la muestra representativa en cada una de las Escuela de la Universidad

Por otro lado, sería interesante tener en cuenta otras variables que puedan influir en las actitudes de los estudiantes sin discapacidades hacia sus compañeros con algún tipo de discapacidad en el aula de estudio como: la procedencia de los estudiantes, sus creencias religiosas, el nivel socioeconómico... De este modo, en el caso de que se encontraran otros factores con una influencia significativa en las actitudes hacia esta población, como pueden ser los medios de comunicación, se podría plantear una intervención que vaya más allá de la formación académica, abarcando así a una mayor parte de la población.

Además, se debería considerar la posibilidad de valorar las actitudes mostradas por los estudiantes de otras universidades, con el fin de comparar los datos obtenidos y comprobar si pueden generalizarse los resultados de la investigación a otra población.

\section{Conclusiones}

A partir de la presentación y del análisis de resultados, se generaron un conjunto de conclusiones referentes a la actitud de individuos sin discapacidad hacia las personas con discapacidad cuidando las limitaciones que presenta el 
estudio, pues la muestra de individuos no resulta representativa para proyectar las conclusiones a la población total de individuos que estudian con personas con discapacidad.

Es primordial para el correcto desempeño y una óptima realización de las actividades académicas de una persona con discapacidad, que su entorno inmediato y social lo acepte, esto se logra, entre otras cosas, teniendo una actitud favorable hacia ellos.

\section{Recomendaciones}

Esta investigación, a pesar de alcanzar los objetivos planteados, presenta algunos inconvenientes, uno de ellos es que los resultados arrojados en este estudio no se pueden generalizar debido a que sólo se tomó una muestra del total de los estudiantes sin discapacidad que estudian en el Centro Regional de Ibagué de la UNAD, sin embargo el estudio abre camino a futuras investigaciones de ésta índole, por tal razón es importante considerar realizar una réplica del ejercicio a nivel de la zona Sur donde pertenece el centro regional Ibagué y en las otras zonas de UNAD, con el fin de conocer a mayor escala la actitud de las personas hacia la discapacidad en escenarios educativos.

Realizar investigaciones donde se mida tanto la actitud de las personas que estudian con compañeros con discapacidad, como la percepción que tienen estos últimos hacia su ambiente inmediato, con la finalidad de comprobar que la actitud se corresponde con lo que perciben las personas con discapacidad en su ámbito educativo.

Caracterizar la población con el fin de conocer edades, género, estrato, ubicación, y nivel socio económico entre otros, que tienen las personas con discapacidad en los Centros Regionales donde están incluidas, y qué tipo de discapacidad presentan, con el propósito de conocer qué funciones y niveles de responsabilidades tienen.

Se recomienda realizar este estudio a mayor escala, tomando en cuenta una población grande de estudiantes que tenga compañeros con personas con discapacidad. Así mismo se invita a futuros investigadores a tomar en cuenta el grado de discapacidad que tengan las personas que están incluidas en los Centros Regionales de la UNAD, ya que podrían existir diferencias en la actitud de los estudiantes dependiendo del grado de discapacidad que tengan las personas. 


\section{Referencias bibliográficas}

Abreu ; Álvarez y Varganciano. 1998. El Proceso de Inserción Laboral de dos Personas con Discapacidad Intelectual Pertenecientes al Programa de Empleo con Soporte Creando Independencia de ASODECO. Trabajo especial de grado, Caracas: Instituto Universitario AVEPANE.

Alomar, E y Cabré, M. 2005. El trabajo de jóvenes con discapacidad intelectual en entornos normalizados. Revista Síndrome de Down, 22: 118-124.

Aramayo, M (2005). Universidad y diversidad. Venezuela: hacia una educación superior de calidad para las personas con discapacidad. Caracas: Universidad Central de Venezuela.

Beckles, B. (2004). Pobreza y Discapacidad: Abogando para eliminar la exclusión social. Seminario: Discapacidad y reducción de la pobreza: incluyendo la discapacidad en la agenda de desarrollo (experiencias de Asia y América Latina y El Caribe), Trinidad y Tobago, Banco Interamericano de Desarrollo.

Cantero, F.J.; León, J.M. y Barriga, S. 1998. Actitudes: naturaleza, formación y cambio. En J.M. León y otros (Coords.). Psicología Social. Orientaciones teóricas y ejercicios prácticos. Pp. 117-132.Madrid: McGraw-Hill.

Dávila, S. 1999. La evaluación de valores y actitudes. Revista de Educación y Cognición. (Recuperado 6 de noviembre de 2012) http//nalenjandria.com.ar/akademeia/ gmc/04EDU.htm

Dugarte Ceballos, E. B. 2007. Actitudes que se involucran en el proceso de integración socio laboral de las personas con discapacidad intelectual, Memoria de Grado, Caracas: Universidad Católica Andrés Bello.

Fournier, M.V. 2008. Evaluación de actitudes discriminativas y prejuicios hacia diversas poblaciones, con estudiantes universitarios de la gran área metropolitana de San José, Costa Rica. San José, Costa Rica: Centro de Investigación y Promoción para América Central de Derechos Humanos.

García, C. E. 2004. Conceptos y términos sobre la discapacidad de la OMS [Artículo en línea]. Consultado el día 2 de Noviembre de 2008. http://usuarios.discapnet.es/ disweb2000/Portadas/13jun2004.htm

Gómez, M. M. 2006. Introducción a la metodología de la investigación científica. Córdoba: Editorial Brujas.

Guitián, I. y Rojas, S. 2002. La incorporación de un adulto con retardo mental al campo laboral. Trabajo especial de grado, Caracas: Instituto Universitario AVEPANE. 
Hernández Sampieri, R., Fernández, C., y Baptista, P. 2006. Metodología de la investigación, México: Editorial Mc Graw Hill.

Infante, M. y Gómez, V. 2004. Actitudes de los estudiantes de educación hacia la integración de personas con discapacidad y hacia la educación multicultural. $C \& E$ : Cultura y Educación. 16(4): 371-384.

Jacay, S. 2004. Los derechos de las personas con discapacidad: un balance de su protección en los Sistemas Internacionales de Protección de los Derechos Humanos y en los países de la Región Andina, Lima: Comisión Andina de Juristas.

Mella Díaz, S.; González Quiroga, L. 2007. Actitudes de los estudiantes de la salud de la Universidad de Chile hacia las personas con discapacidad. Revista Chilena de Terapia Ocupacional, 7: 1-14.

Ministerio de Educación Nacional (MEN). 2006. Ministerio de Educación Nacional, (recuperado 15 de noviembre de 2010). http://www.mineducacion.gov.co/1621/w3article-231372.html

Ministerio de las Tecnologías (MINTIC). 2009. NTC 5854. Accesibilidad a Páginas Web. Instituto Colombiano de Normas Técnicas y Certificación, ICONTEC (Recuperado 15 de Junio 2011), http://www.lalibreriadelau.com/libros-de-medicina-ca30_42/ntc-normatecnica-colombiana-ntc-5854-accesibilidad-paginas-p150120.

Moya, M. y Ruíz, J. 1996. Las actitudes: marco teórico. En F.J. Perales; J. Gutiérrez y P. Álvarez (Eds.) I Jornadas sobre actitudes y educación ambiental. Pp. 2-38. Granada: I.C.E.

Muñoz, C. 2005. Integración social de personas con discapacidad. Curso de formación general, Santiago de Chile: Universidad de Chile.

Ramírez, R. 2008. Estadística de matrícula de estudiantes con alguna condición de discapacidad. Bogotá: Universidad Nacional Abierta y a Distancia - UNAD.

Rodríguez, A. 1996. Psicología social de los prejuicios. En J.L. Álvaro; A. Garrido y J.R. 1996.

Shakespeare, R. 1981. La psicología de la invalidez, México: Editorial Continental.

Sierra Bravo, R. 1994. Técnicas de investigación social: teoría y ejercicios, Madrid: Editorial Paraninfo S.A.

Tejeiro, A. y Ortiz, P. 1998. Actitudes sociales hacia la discapacidad física. En: Mp. Sánchez y M.A. Quiroga (coords.). Perspectivas Actuales en la Investigación Psicológica de las Diferencias Individuales, Pp. 260-265. Madrid: Centro de Estudios Ramón Areces.

Verdugo, M. A.; Arias, B., y Jenaro, C. (1994): Actitudes hacia las personas con minusvalía. Madrid, Ministerio de Asuntos Sociales, Instituto Nacional de Servicios Sociales. 